PROCEEDINGS OF THE

AMERICAN MATHEMATICAL SOCIETY

Volume 128, Number 5, Pages 1567-1569

S 0002-9939(00)05695-1

Article electronically published on February 7, 2000

\title{
STABILITY OF $n$-VORTICES IN THE GINZBURG-LANDAU EQUATION
}

\author{
JAMES COLEMAN
}

(Communicated by David S. Tartakoff)

\begin{abstract}
We consider the class of $n$-vortex solutions to the time-independent Ginzburg-Landau equation on $\mathbf{R}^{2}$. We prove an inequality governing the solutions of a particular boundary value problem. This inequality is crucial for an elementary proof by Ovchinnikov and Sigal that such $n$-vortices are unstable in the case $|n| \geq 2$.
\end{abstract}

We consider here the time-independent Ginzburg-Landau equation

$$
\Delta \varphi+\left(1-|\varphi|^{2}\right) \varphi=0,
$$

where $\varphi: \mathbf{R}^{2} \rightarrow \mathbf{C}$ satisfies the boundary condition $|\varphi(r, \theta)| \rightarrow 1$ as $r \rightarrow \infty$. The simplest family of solutions of (1) are the $n$-vortices [1] where $n$ is an arbitrary integer. They are defined by $\varphi_{n}(r, \theta)=e^{i n \theta} f_{n}(r)$, where $f_{n}:[0, \infty) \rightarrow \mathbf{R}$ satisfies

$$
f_{n}^{\prime \prime}(r)+\frac{1}{r} f_{n}^{\prime}(r)+\left(1-\frac{n^{2}}{r^{2}}\right) f_{n}(r)-f_{n}^{3}(r)=0, \quad f_{n}(0)=0, \quad f_{n}(\infty)=1 .
$$

The existence and uniqueness of a solution for (2) are shown in [2, 4].

Of particular interest is the question of the stability of $n$-vortices under small perturbations, originally studied by Hagan [3]. Later, it was shown in [7] using variational methods that $n$-vortices are unstable for $|n| \geq 2$. An alternative, elementary proof was proposed by Ovchinnikov and Sigal [5] and makes use of an inequality whose validity was only checked experimentally. In this note, we present a simple proof of this inequality, as follows.

Proposition. Let $f_{n}$ be the solution of (2) with $|n| \geq 2$. Then $f_{n} \leq f_{n}^{\max }$, where

$$
f_{n}^{\max }(r)=\frac{r^{2}}{r^{2}+\frac{n^{2}}{2}}, \quad r \in[0, \infty) .
$$

For the proof, we recall the following comparison result for ODE's [6].

Lemma. Let $(a, b)$ be an interval in $\mathbf{R}$, let $\Omega=\mathbf{R}^{2} \times(a, b)$, and let $H \in C^{1}(\Omega, \mathbf{R})$. Suppose $f \in C^{2}(a, b)$ satisfies $f^{\prime \prime}+H\left(f, f^{\prime}, r\right)=0$. If $H_{f} \leq 0$ on $\Omega$ and if there exist functions $M, m \in C^{2}(a, b)$ satisfying $M^{\prime \prime}(r)+H\left(M(r), M^{\prime}(r), r\right) \leq 0$ and $m^{\prime \prime}(r)+$ $H\left(m(r), m^{\prime}(r), r\right) \geq 0$, as well as the boundary conditions $m(a) \leq f(a) \leq M(a)$ and $m(b) \leq f(b) \leq M(b)$, then for all $r \in(a, b)$ we have $m(r) \leq f(r) \leq M(r)$.

Received by the editors April 15, 1999.

2000 Mathematics Subject Classification. Primary 35Q55.

(C)2000 American Mathematical Society 
Proof of the Proposition. We apply the lemma to our problem, taking $a=0, b=$ $\infty, M=f_{n}^{\max }, m=f_{n}$, and

$$
H\left(f_{n}, f_{n}^{\prime}, r\right)=\frac{1}{r} f_{n}^{\prime}+\left(1-\frac{n^{2}}{r^{2}}\right) f_{n}-f_{n}^{3} .
$$

We easily check that all the conditions of the lemma are satisfied, except possibly $H_{f_{n}} \leq 0$. Now,

$$
H_{f_{n}}\left(f_{n}, f_{n}^{\prime}, r\right)=\left(1-\frac{n^{2}}{r^{2}}\right)-3 f_{n}^{2},
$$

and so $H_{f_{n}} \leq 0$ as long as $f_{n}$ lies above the curve $g_{n}$ given by

$$
g_{n}(r)= \begin{cases}0, & 0 \leq r \leq n ; \\ \sqrt{\frac{1}{3}\left(1-\frac{n^{2}}{r^{2}}\right),} & r \geq n .\end{cases}
$$

Let $U=\left\{r \in(0, \infty), f_{n}(r)>g_{n}(r)\right\}$. If $U=(0, \infty)$, then the theorem follows immediately. Otherwise, since $U$ is open it consists of countably many disjoint open intervals. If $(c, d)$ is any such interval, then applying the lemma to $(c, d)$ we have $f_{n} \leq f_{n}^{\max }$ on all of $(c, d)$. Hence the inequality holds on $U$. But it is easily checked that $g_{n}(r) \leq f_{n}^{\max }(r)$ for all $r \in(0, \infty)$, and so $f_{n} \leq f_{n}^{\max }$ for all $r \in[0, \infty)$.

With this inequality in place, the proof of instability of $n$-vortices for $|n| \geq 2$ proceeds along the lines given in [5]. Begin by introducing the renormalized energy functional $\mathcal{E}$, defined by

$$
\mathcal{E}(\varphi)=\frac{1}{2} \int_{\mathbf{R}^{2}}\left(|\nabla \varphi(x)|^{2}-\frac{\operatorname{deg}(\varphi)^{2}}{|x|^{2}} \chi(x)+\frac{1}{2}\left(|\varphi(x)|^{2}-1\right)^{2}\right) d^{2} x,
$$

where $\chi$ is a smooth weight function inserted to make the integral finite, and $\operatorname{deg}(\varphi)$ is the degree of the function $\varphi$ (in particular, $\operatorname{deg}\left(\varphi_{n}\right)=n$ ). We will work with the space $X$ defined by $X=\left\{\varphi \in H^{1}\left(\mathbf{R}^{2}\right), \mathcal{E}(\varphi)<\infty\right\}$. The tangent space $T_{\varphi} X$ to $X$ at the point $\varphi$ will be identified with the Sobolev space $H^{1}\left(\mathbf{R}^{2}\right)$.

A function $\varphi$ is a solution of (11) if and only if it is a critical point of the energy functional (7). Our goal is to determine whether a given solution is an extremum or a saddle point of this functional. This can be done by considering the usual first and second variations [8] $d \mathcal{E}_{\varphi}: T_{\varphi} X \rightarrow \mathbf{R}$ and $d^{2} \mathcal{E}_{\varphi}: T_{\varphi} X \times T_{\varphi} X \rightarrow \mathbf{R}$. To prove the instability of a solution $\varphi$ it suffices to find a $u \in T_{\varphi} X$ such that $d^{2} \mathcal{E}_{\varphi}(u, u)<0$.

Now, we claim that $u(r, \theta)=e^{-r^{2} / 4}$ satisfies this requirement as long as $|n| \geq 2$. Using the fact that $f_{n} \leq f_{n}^{\max }$, we have

$$
\begin{aligned}
& d^{2} \mathcal{E}_{\varphi_{n}}(u, u)=\operatorname{Re} \int_{\mathbf{R}^{2}} \bar{u}\left(-\Delta u+\left(2\left|\varphi_{n}\right|^{2}-1\right) u+\varphi_{n}^{2} \bar{u}\right) \\
& =\operatorname{Re} \int_{0}^{2 \pi} \int_{0}^{\infty} e^{-r^{2} / 4}\left(-\Delta\left(e^{-r^{2} / 4}\right)+\left(2 f_{n}^{2}-1\right) e^{-r^{2} / 4}+f_{n}^{2} e^{2 i n \theta} e^{-r^{2} / 4}\right) r d r d \theta \\
& \leq-\frac{\pi}{2} \int_{0}^{\infty} \frac{r^{3}\left[\left(2 r^{2}+n^{2}-8\right)^{2}+16 n^{2}-64\right] e^{-r^{2} / 2}}{\left(2 r^{2}+n^{2}\right)^{2}} d r .
\end{aligned}
$$

For $|n| \geq 2$ this expression is clearly negative, and the result follows immediately. 


\section{REFERENCES}

[1] F. Bethuel, H. Brézis and F. Hélein, Ginzburg-Landau Vortices, Birkhauser, 1994. MR 95c:58044

[2] X. Chen, C.M. Elliott and T. Qi, Shooting method for vortex solutions of a complex-valued Ginzburg-Landau equation, Proc. Roy. Soc. Edinburgh Sect. A. 124 (1994), no. 6, 1075-1088. MR 95j:35208

[3] P. Hagan, Spiral waves in reaction-diffusion equations, SIAM J. of Appl. Math. 42 (1982), no. 4, 762-786. MR 84c:92069

[4] R.M. Hervé and M. Hervé, Etude qualitative des solutions réelles d'une équation différentielle liée à l'équation de Ginzburg-Landau, Ann. Inst. H. Poincaré Anal. Non Linéaire 11 (1994), no. 4, 427-440. MR 95g:35194

[5] Y.M. Ovchinnikov and I.M. Sigal, Ginzburg-Landau equation I. Static vortices, Partial Differential Equations and their Applications, 199-220, CRM Publications, 1997. MR 98k:35179

[6] M.H. Protter and H.F. Weinberger, Maximum Principles in Differential Equations, Springer, 1984. MR 86f:35034

[7] I. Shafrir, Remarks on solutions of $-\Delta u=\left(1-|u|^{2}\right) u$ in $\mathbf{R}^{2}$, C.R. Acad. Sci. Paris Sér I Math. 318 (1994), no. 4, 327-331. MR 95c:35091

[8] M. Struwe, Variational Methods, Springer, 1990. MR 92b:49002

Department of Mathematics, University of Toronto, Toronto, Ontario, Canada M5S 3G3

E-mail address: coleman@math.utoronto.ca 